\title{
Current Status of RENO Experiment
}

\author{
Soo-Bong Kim (for the RENO collaboration) \\ KNRC, Dept. of Physics and Astronomy, Seoul National University \\ Sillim-dong, Gwanak-gu, Seoul 151-742, KOREA \\ E-mail:sbkim@phya.snu.ac.kr
}

The RENO(Reactor Experiment for Neutrino Oscillation) experiment is under construction to measure the value of the smallest and unknown neutrino mixing angle $\theta_{13}$. The experiment will compare the measured fluxes of electron antineutrinos at two detectors located at $290 \mathrm{~m}$ and 1.4 $\mathrm{km}$ distances from the center of the Yonggwang nuclear reactors in Korea. The detectors are near completion, and data-taking is expected to start in early 2011. An estimated systematic uncertainty associated with the measurement is less than $0.6 \%$, and an expected statistical error is about $0.3 \%$. With three years of data, the experiment will search for the mixing angle values of $\sin ^{2} 2 \theta_{13}$ down to 0.02 in the $90 \%$ C.L. limit. In this talk, the construction status is presented.

35th International Conference of High Energy Physics (ICHEP2010)

Paris,France

July 22-28, 2010

\section{Introduction}

The current best limit on the last unknown mixing angle $\theta_{13}$ is given by the Chooz reactor neutrino experiment.[1] A high precision measurement of reactor neutrino oscillation can be achieved by multiple detectors because experimental sensitivity would be nearly unaffected by the uncertainties related to antineutrino flux and detection [2]. The RENO experiment will try to measure $\theta_{13}$ or at least improve the current constraint using reactor neutrinos.

There are degeneracies and correlations among oscillation parameters in future accelerator based long baseline neutrino experiments whereas reactor neutrino experiments provide a clean information on $\sin ^{2} 2 \theta_{13}$. A measurement of or stringent limit on $\theta_{13}$ would strongly motivate the investment for a long-term program to measure $\mathrm{CP}$ violation parameters at accelerators.

\section{The RENO Experiment}

\subsection{Yonggwang Nuclear Reactors}

The Yonggwang nuclear power plant is located in the west coast of southern part of Korea, about $400 \mathrm{~km}$ from Seoul. The power plant consists of six reactors producing total thermal power of $16.4 \mathrm{GW}$, the second largest in the world. The six reactors are lined up in roughly equal 
distances and span $\sim 1.3 \mathrm{~km}$.

\subsection{Near and Far Detectors}

The experimental setup consists of two identical detectors of 16-ton Gadolinium loaded liquid scintillator target, located near and far from the reactor array to measure the deviations of the observed flux from the inverse square distance law. The near and far detectors are to be placed roughly $290 \mathrm{~m}$ and $1.4 \mathrm{~km}$ away from the center of the reactor array, respectively. The near detector is to be located at underground of a $70 \mathrm{~m}$ high hill with an overburden of $\sim 110$ mwe and the far detector at underground of a $260 \mathrm{~m}$ high mountain with an overburden $\sim 450$ mwe.

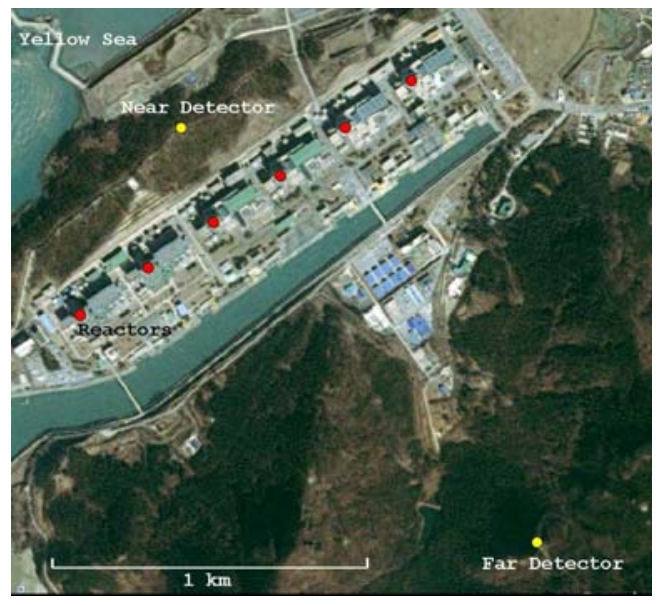

Figure 1. Layout of the RENO experimental site. Red dots and yellow dots represent reactors and detectors, respectively. Six reactors are equally spaced in a $1280 \mathrm{~m}$ span.

\subsection{Status}

Consideration of the RENO experiment began in early 2004 based on White Paper for a New Nuclear Reactor Neutrino Experiment,[3] and the construction started in March 2006. The KHNP, the company operating the Yonggwang nuclear power plant, has allowed us to carry out the experiment in the restricted area. The local government and residents have also expressed their best cooperation for RENO. Geological survey and tunnel design were completed in 2007. Civil construction for underground facility, including excavation of two tunnels, began in middle 2008 and was completed in early 2009. Construction of both near and far detectors is expected to be completed by the end of 2010. Data-taking is planned to start in early 2011.

\section{The RENO Detector}

The RENO detector, having a cylindrical shape of $8.8 \mathrm{~m}$ in height and $8.4 \mathrm{~m}$ in diameter, consists of a neutrino target, a gamma catcher, a buffer and a veto.[4]

\subsection{Neutrino Target}

The neutrino target consists of $0.1 \%$ Gd loaded liquid scintillator in a cylindrical acrylic container of $140 \mathrm{~cm}$ in radius, $320 \mathrm{~cm}$ in height, and $25 \mathrm{~mm}$ in thickness. It has a total volume of $19.2 \mathrm{~m}^{3}$ and a target mass of 16.1 tons. 


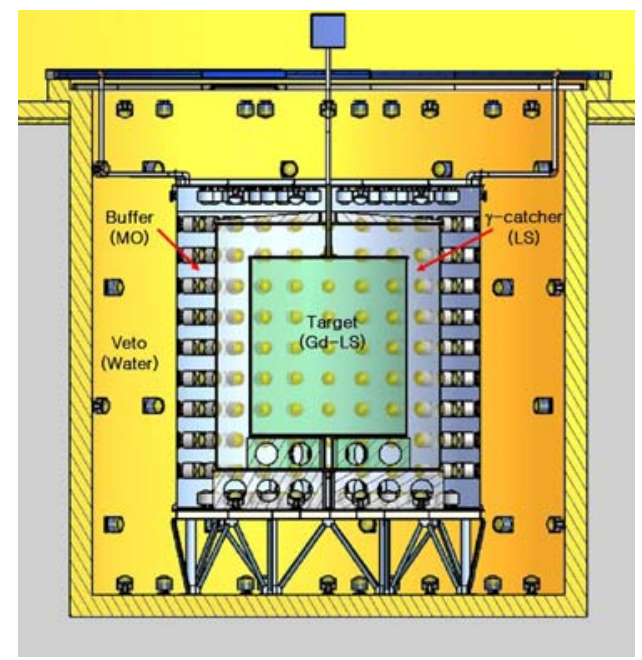

Figure 2. A schematic view of RENO detector.

\subsection{Gamma Catcher}

Gamma catcher surrounds the neutrino target with $60 \mathrm{~cm}$ thick unloaded liquid scintillator of $33.2 \mathrm{~m}^{3}$ in volume and 28.6 tons in mass. The gamma catcher is contained in a cylindrical acrylic vessel of $200 \mathrm{~cm}$ in radius, $440 \mathrm{~cm}$ in height, and $30 \mathrm{~mm}$ in thickness.

\subsection{Buffer}

A $70 \mathrm{~cm}$ thick non-scintillating liquid surrounds the gamma catcher to reduce accidental backgrounds coming from outside, mainly from radioactivity in the photomultiplier tubes, by almost two orders of magnitude. A total 64.2 tons of mineral oil is contained in a stainless steel vessel of $270 \mathrm{~cm}$ in radius, $580 \mathrm{~cm}$ in height, and $6 \mathrm{~mm}$ in thickness. This buffer is necessary for keeping the single rate below $10 \mathrm{~Hz}$ in the neutrino target and gamma catcher regions.

\subsection{Acrylic Vessels}

The acrylic vessels should contain aromatic liquids without leak and changing properties for a long-term period, roughly more than 10 years. They should not develop any chemical reaction with the scintillating liquids of neutrino target and gamma catcher, and with nonscintillating mineral oil of buffer for a long time period.

\subsection{Photomultiplier Tube}

A total 354 of 10-inch PMTs in a uniform array are mounted from the inner surface of the buffer vessel, providing $14 \%$ photo-sensitive surface coverage. The cylindrical steel vessel optically isolates the inner detector part from the outer veto system.

\subsection{Veto}

A $1.5 \mathrm{~m}$ thick water layer of 352.6 tons surrounds the whole inner detector with a mass of 110 tons. A total 67 of 10-inch PMTs are mounted in front of Tyvek reflector on a cylindrical steel tank. 


\subsection{Liquid Scintillator}

The neutrino target will be filled with $1 \mathrm{~g} / \mathrm{l}$ Gd loaded liquid scintillator. For Gd loading, carboxylic acids (CBX) is chosen as the basis for ligands to guarantee long-term stability. Linear Alkyl Benzene (LAB) is used as scintillator solvent to replace dodecane and pseudocumene admixture. LAB has a high flash-point of $130^{\circ} \mathrm{C}$ and a good optical property, and is commercially available in Korea.

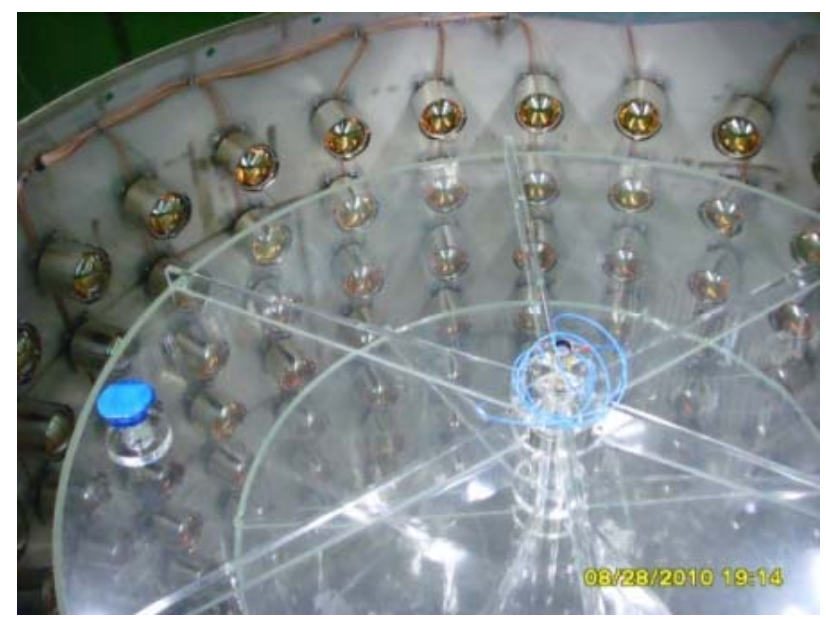

Figure 3. View of detector interior under PMT installation on the buffer vessel walls. Two acrylic vessels are shown with two chimneys for source calibration.

\section{Sensitivity}

RENO is expected to improve the sensitivity on $\sin ^{2} 2 \theta_{13}$ by an order magnitude relative to the current best limit. This will be achieved by reducing both statistical and systematic errors, less than $0.6 \%$. The reactor antineutrinos are detected via the capture on a free proton, emitting a positron and a neutron, with an energy threshold of $1.8 \mathrm{MeV}$. The signature of a neutrino event is a prompt signal of positron followed by the neutron capture on Gd creating gammas with about $8 \mathrm{MeV}$. An expected number of observed antineutrino is roughly 1,300 per day and roughly 110 per day in the near detector and far detector, respectively. An estimated systematic uncertainty associated with the measurement is less than $0.6 \%$. Based on three years of data, it would be sensitive to measure the neutrino mixing angle in the range of $\sin ^{2} 2 \theta_{13}>0.02$. This sensitivity is five times better than the current limit obtained by Chooz.

\section{References}

[1] M. Apollonio et al. (Chooz Collaboration), Eur. Phys. J. C 27, 331 \{2003\}.

[2] L. A. Mikaelyan and V. V. Sinev, Phys. Atomic Nucl. 63, 1002 \{2000\}.

[3] K. Anderson et al., White paper report on using nuclear reactors to search for a value of $\theta_{13}$, [hepex/0402041] (2004).

[4] J.K. Ahn et al., RENO Proposal, [hep-ex/1003.1391] (2010). 